\section{Hidden coeliac disease in patients suffering from oral lichen planus}

Editor

Coeliac disease (CD) is a chronic immune-mediated disease, triggered by gluten ingestion in genetically susceptible subjects resulting in small intestinal mucosal injury with subsequent nutrient malabsorption. ${ }^{1} \mathrm{CD}$ is a disease with a heterogeneous and often unsuspected range of clinical presentations; its 'typical' presentation, characterized by a gastrointestinal manifestations, is less frequent and many CD patients show 'atypical' - non-gastroenterological - signs, as some oral lesions (e.g. recurrent aphthous stomatitis, dental enamel defects and unspecific atrophic glossitis). ${ }^{1}$ In this regard, few studies have analysed the association of $\mathrm{CD}$ and oral lichen planus (OLP). ${ }^{2-5}$ In this study, we assessed the frequency of unrevealed CD among OLP patients and the possible effects of a gluten-free diet (GFD) on OLP lesions in CD patients.

Twenty-three patients with clinically and histologically confirmed OLP, receiving topical corticosteroid treatment underwent blood samples to assess full blood count, serum folate, vitamin $\mathrm{B} 12$, iron and CD antibodies levels.

Two female patients, without any further systemic signs and symptoms were found to have positive CD antibodies associated with haematinic deficiencies, underwent small intestinal biopsy arising the diagnosis of CD and were revaluated after 6 months of GFD. They presented atrophic/erosive OLP localized on the buccal mucosa (associated with a burning sensation despite topical corticosteroid therapy).

Six months after GFD, a normalization of serum levels of folate, vitamin B12 and iron, accompanied by an improvement of the oral soreness and of the atrophic/erosive areas, were observed in both patients.

OLP is a chronic disease affecting the oral mucosa, having a T-cell-mediated autoimmune pathogenesis and an unknown aetiology in most cases. ${ }^{6}$ It may be associated with a number of immune-mediated systemic disorders, although this association could be merely accidental and not linked to the autoimmune pathogenesis. $^{7}$

Despite their similar autoimmune pathogenesis and the findings of the association between CD and oral manifestations, ${ }^{1}$ few stud-

All the Authors have declared that they neither have potential competing interests nor received financial support for this study. ies, often with conflicting results, have assessed the association between CD in OLP patients.

The first association between these two conditions was reported $^{2}$ in the case of a 70-year-old male with a biopsy-proven erosive OLP. Iron, folate and vitamin B12 deficiencies indicated a jejunal biopsy, which demonstrated the presence of CD. Surprisingly, GFD was found to relieve OLP within six months. The hypothesis of an association between CD and OLP has been promptly refuted by Scully et al., ${ }^{3}$ who did not diagnosed CD in none of the 103 OLP patients investigated, concluding that their association could be only accidental. However, another study revealed that of the 39 OLP patients recruited, 22 showed positive CD antibodies. ${ }^{4}$ Finally, a case of a 9-year-old women with erosive lichen planus associated with hyper IgE syndrome and CD was also described. ${ }^{5}$

Our results, obtained from a small sample size, do not allow us to confirm any pathogenic association between CD and OLP, but they represent an interesting observation.

Some atrophic/erosive and ulcerative oral lesions may be associated with underlying haematinic deficiencies, ${ }^{8,9}$ which could characterize the nutrient malabsorption status of $\mathrm{CD}$ patients. Both vitamin B12 and folic acid favour tissue tropism, principally acting on all tissues, as the oral epithelium, with a cell turnover promoting their regeneration. ${ }^{10}$ Therefore, the haematinic deficiency in our patients may negatively influence the rapidly dividing mucosal cells affected by a pre-existing OLP. The resolution of the burning sensation, the amelioration of the atrophic/erosive areas and haematinic level normalization after GFD, observed in our patients with OLP refractory to local therapy, support the hypothesis of a cause-effect correlation between haematinic deficiencies and the soreness associated with atrophic/erosive oral lesions.

In conclusion, as an improvement in nutritional status leads to relief in oral soreness as opposed to the often less successful palliative medications, we suggest the search for possible nutritional deficiencies in any patient with atrophic/erosive oral lesions, including OLP, and subsequently to include a screening for CD.

D. Compilato, ${ }^{\dagger}$ A. Carroccio, ${ }^{\ddagger}$ G. Campisi ${ }^{\dagger, *}$

'Department of Surgical and Oncological Disciplines 'G. Messina', Unit of Oral Medicine, University Hospital of Palermo, Palermo, Italy ‡Operative Unit of Medicine, A.O. Ospedali Riuniti di Sciacca (AG),

Sciacca, Italy

${ }^{*}$ Correspondence: G. Campisi.

E-mail: campisi@odonto.unipa.it 


\section{References}

1 Pastore L, Carroccio A, Compilato D et al. Oral manifestations of celiac disease. J Clin Gastroenterol 2008; 42: 224-232.

2 Fortune F, Buchanan JA. Oral lichen planus and coeliac disease. Lancet 1993; 341: 1154-1155.

3 Scully C, Porter SR, Eveson JW. Oral lichen planus and coeliac disease. Lancet 1993; 341: 1660.

4 Jokinen J, Peters U, Maki M et al. Celiac sprue in patients with chronic oral mucosal symptoms. J Clin Gastroenterol 1998; 26: 23-26.

5 Ruiz Villaverde R, Blasco Melguizo J, Menendez Garcia Estrada A, Diez Garcia F. Erosive mucosal lichen associated to hyper IgE syndrome and coeliac disease. An Pediatr (Barc) 2004; 60: 281-282.

6 Scully C, Carrozzo M. Oral mucosal disease: Lichen planus. $\mathrm{Br} J$ Oral Maxillofac Surg 2008; 46: 15-21.

7 Compilato D, Paderni C, Di Fede O et al. Association of oral lichen planus with thyroid disease in a Finnish population: A retrospective case-control study: 'different finding from a Mediterranean area'. Oral Surg Oral Med Oral Pathol Oral Radiol Endod 2011; 111: 12-13.

8 Thongprasom K, Youngnak P, Aneksuk V. Folate and vitamin B12 levels in patients with oral lichen planus, stomatitis or glossitis. Southeast Asian J Trop Med Public Health 2001; 32: 643-647.

9 Compilato D, Carroccio A, Calvino F et al. Haematological deficiencies in patients with recurrent aphthosis. J Eur Acad Dermatol Venereol 2010; 24: 667-673.

10 Thomas DM, Mirowski GW. Nutrition and oral mucosal diseases. Clin Dermatol 2010; 28: 426-431.

DOI: 10.1111/j.1468-3083.2011.04054.x

\section{A shortened docetaxel desensitization protocol for use in special cases}

Editor

We report a successful shortened docetaxel desensitization protocol in a patient with docetaxel hypersensitivity.

A 62-year-old male Caucasian patient presented with a metastasized carcinoma of the prostate, which had been diagnosed 5 years ago. Treatment with anti-androgen therapy by the department of urology initially led to stable prostate specific antigen (PSA) levels and stopped growth of metastases.

When PSA-levels and metastatic growth increased again, the patient received a docetaxel chemotherapy. Docetaxel was administered with $75 \mathrm{mg} / \mathrm{m}^{2}$ body surface. The first infusion was well tolerated, except of mild dyspnoea and moderate increase in blood pressure.

The second infusion 4 weeks later had to be stopped after a few minutes due to generalized flush, massive dyspnoea and an associated hypertensive crisis.

The patient was transferred to our department for diagnosis and treatment. A skin prick test with docetaxel $40 \mathrm{mg} / \mathrm{mL}$ in physiological saline was negative. An intradermal test showed a positive reaction only at $0.4 \mathrm{mg} / \mathrm{mL}$, but not at $0.04 \mathrm{mg} / \mathrm{mL}$, which is rather compatible with an irritative than an allergic skin test reaction (Fig. 1). ${ }^{1}$

Premedication included oral glucocorticosteroids 12 and $6 \mathrm{~h}$ before and i.v. H1-antihistamines in $500 \mathrm{~mL}$ sodium chloride $30 \mathrm{~min}$ before the therapy.

Emergency back up was warranted by electrocardiogram-, oxygen- and blood pressure monitoring, emergency medication availability, venous access, oxygen bottle and mask and supervision by medical staff.

After clinical and laboratory assessment of the patient, the first desensitization-infusion was initiated, following the $5.8 \mathrm{~h}$ paclitaxel desensitization scheme by Feldweg et al. ${ }^{2}$ (Table 1). This was tolerated very well, as was the second infusion 4 weeks later. No immediate or delayed hypersensitivity reaction was observed.

For practical reasons, the third and fourth infusions were administered according to a shortened $4.8 \mathrm{~h}$ desensitization scheme (Table 2). Later on, this scheme has been used for the remaining infusions without adverse events.

\section{Comments}

Docetaxel is a well-known cytotoxic agent belonging to the group of taxanes, used in treatment of different cancers of the breast, lung, ovary, pancreas and other tissues. ${ }^{3}$

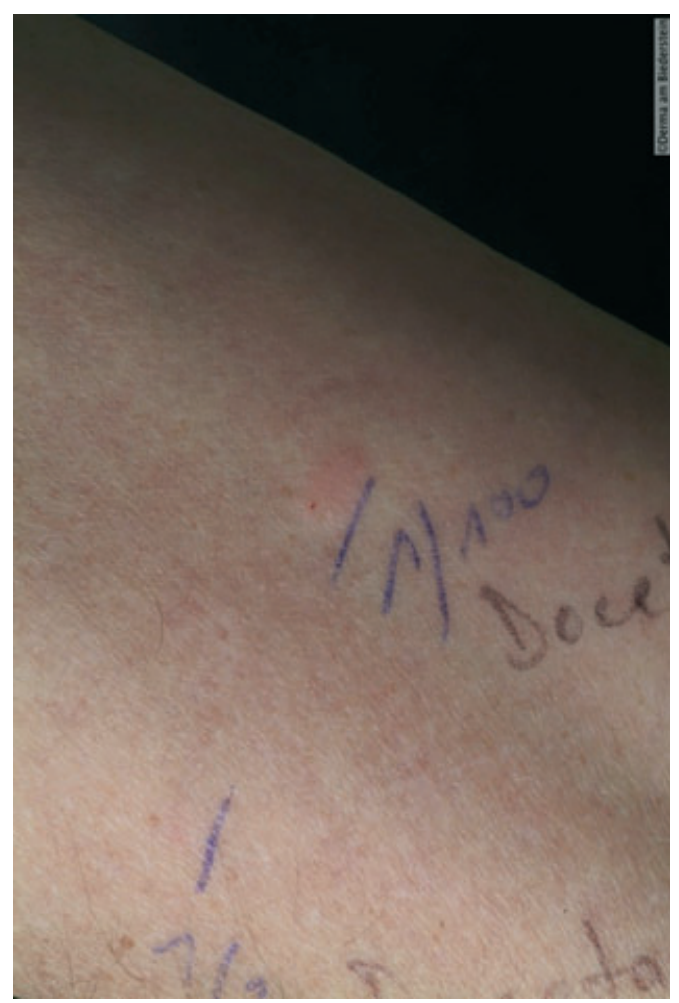

Figure 1 Intradermal test at a level of $1: 100$ diluted docetaxel solution, positive reaction, presumably irritative. 